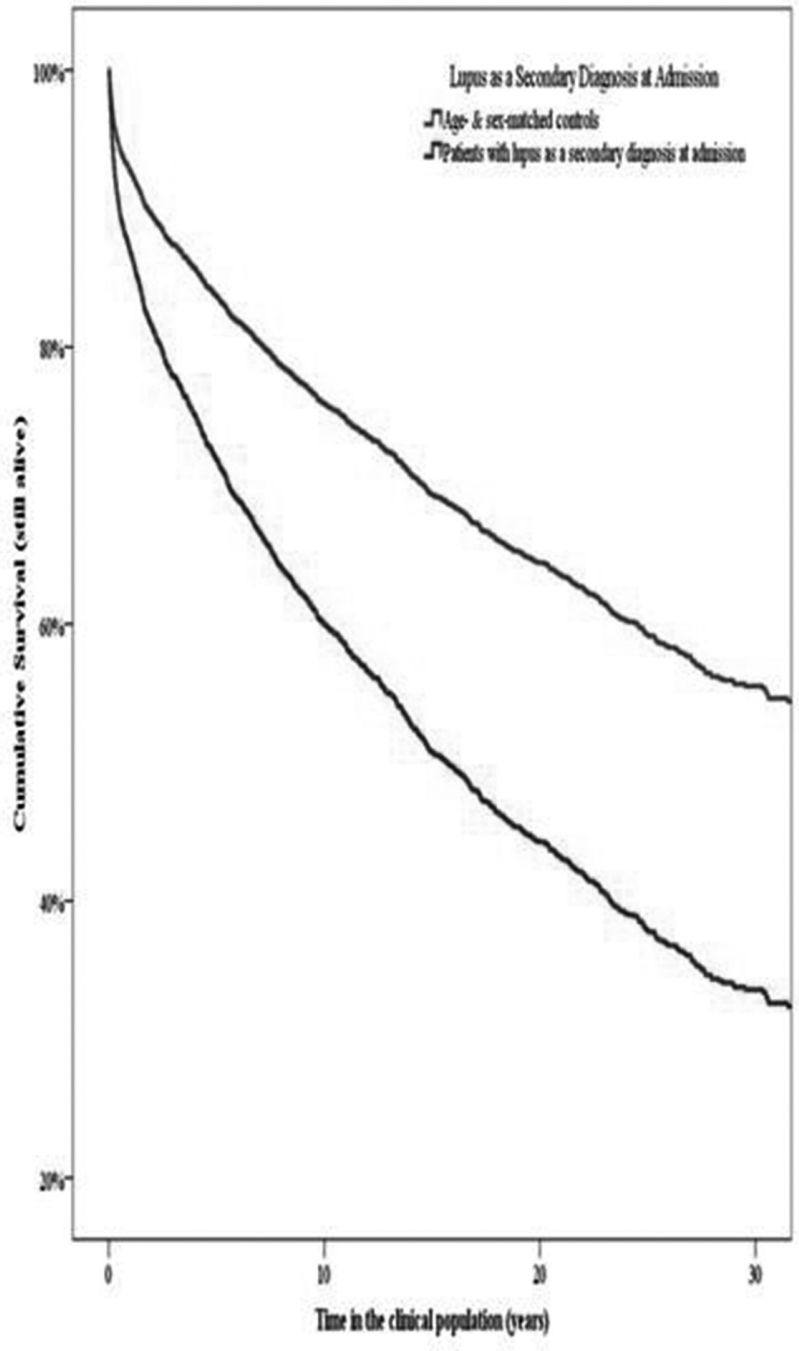

Abstract 420 Figure 1 Kalpan-Meier suvival analysis of mortality outcomes for SLE patients and age- \& sex-matched controls (free of rheumatic disease conditions) from index hospitalisation (Log Rank (Mantel-Cox) $\left.\chi^{2} 158.265, \mathrm{p}<0.001\right)$.

compared to those who delivered later. Preterm delivery could be a surrogate marker for active SLE in pregnancy.

\section{AUTOANTIBODIES PROFILE IN SYSTEMIC LUPUS ERYTHEMATOSUS (SLE) PATIENTS: A STUDY IN MALARIA ENDEMIC AREA}

${ }^{1} \mathrm{R}$ Tripathy*, ${ }^{2} \mathrm{~B}$ Das, ${ }^{2} \mathrm{~S}$ Pattanaik, ${ }^{3} \mathrm{~A}$ Panda. ${ }^{1}$ S. C.B. Medical College, Department of Biochemistry, Cuttack, India; ${ }^{2}$ SCB Medical College, Department of Medicine, Cuttack, India; ${ }^{3}$ Central University of Jharkhand, Centre for Life Sciences, Ranchi, India

\subsection{6/lupus-2017-000215.425}

Background and aims Systemic lupus erythematosus is an autoimmune disorder characterised by elevated levels of autoantibodies. Association of specific autoantibodies with different clinical manifestations of SLE has been documented. Malaria infection is associated with raised levels of autoantibodies. It is not known what happens to autoantibody productions in SLE patients residing in malarial endemic areas and its association with disease manifestation. In the present study,we enrolled SLE patients residing in from different areas of Odisha state, Indiaand investigate possible association of $P$. falciparum endemicity with autoantibodies profile of SLEpatients.

Methods A total of 190 SLE patients from different districts of Odisha, which is endemic for $P$. falciparum malaria were enrolled in the present study. Clinically assessed, and autoantibodies levels were quantified by standard laboratory procedures. They were grouped as 1) Patients from low endemic area based on Annual Parasite Index (API) as low (API <4) and 2) High (API >4) and the data was analysed.

Results The mean age and disease duration of SLE patients were 28.44 and 1.67 years, respectively. 128 patients resided in lower endemic areas and 62 in high endemic areas. 62\%of patients from higher endemic areas had nephritis and other major manifestations like NPSLE and myocarditis. Interestingly, SLE patients from areas(API >4) displayed higher levels of anti-Sm. Other auto antibodies levels were comparable among the two groups.

Conclusions The results of the present study revealed an association of malarial endemicity with differential production of autoantibodies, namely Sm. However, the role of malaria in the pathogenesis of SLE needs to be validated in a prospective study.

\section{RENAL ACTIVITY AND DAMAGE INCUR HIGHEST MEDICAL COSTS AMONG FILIPINO PATIENTS WITH SYSTEMIC LUPUS ERYTHEMATOSUS}

E Venegas*, K Geslani, S Navarra. University of Santo Tomas Hospital, Rheumatology, Manila, Philippines

\subsection{6/lupus-2017-000215.426}

Background and aims Systemic lupus erythematosus (SLE) predominantly affects individuals at peak age of productivity, and medical costs negatively impact on personal, family and community resources. This study aimed to identify annual medical costs and cost predictors among Filipino SLE patients.

Methods Direct annual healthcare costs were determined by survey questionnaires conducted among patients aged $>18$ years with minimum 1 year illness duration, consecutively seen at Lupus Clinics of University of Santo Tomas (UST) Hospital, Manila, Philippines from February to July 2016. Excluded were costs related to biologics. Predictors of cost were estimated using multiple regression analysis.

Results Respondents included 300 SLE patients (93.7\% female) with mean age $32.84 \pm 9.89$ (11-62) at diagnosis, mean disease duration $5.87 \pm 5.58$ (1-36) years. Median and mean annual direct medical cost was Php 90950 and Php 133040 respectively (range Php 17 440-859,050). Annual cost was higher in those requiring dialysis $(n=16)$ compared to nephritis without dialysis $(n=150)$ vs without nephritis ever $(n=134)$, (median Php 595400 vs 144700 vs 55020$), p<0.001$. End stage renal disease (ESRD) $(\mathrm{p}<0.001)$, mycophenolate use $(\mathrm{p}<0.001)$, clinic visits $(\mathrm{p}=0.016)$ and hospitalisation $(p=0.018)$ were independent variables which significantly contributed to annual costs. [Php47.5 = USD1]

Conclusions Nephritis especially if requiring dialysis was the most important cost predictor in this cohort, increasing annual costs up to 7 times. Mycophenolate use, frequency of hospitalisation and clinic visits increased annual costs by $147.2 \%$, $173.8 \%$ and $2.6 \%$ respectively. This study reinforces need for 